\title{
A quarter century of Apoptosis
}

\author{
Arjan W. Griffioen ${ }^{1,3,4} \cdot$ Patrycja Nowak-Sliwinska $a^{2,3,4}$
}

Accepted: 9 April 2021 / Published online: 23 April 2021

(C) The Author(s), under exclusive licence to Springer Science+Business Media, LLC, part of Springer Nature 2021

This year Apoptosis is celebrating its 25th anniversary. Programmed cell death is still gaining interest with an increase to an impressive 97,000 peer-reviewed publications over the last 3 years. Mechanisms of cellular suicide, such as apoptosis, necroptosis, pyroptosis, ferroptosis or autophagy are tightly intermingled in the biology of living organisms and in most cases provide an advantage in an organism's life cycle. These advantages can contribute to sculpting tissues during development, removing tissues when no longer needed or providing heterogeneity. It is probably safe to say that there would be no life without cell death. Therefore, insight in programmed cell death provides understanding in biology in general, as well as in the processes of disease, allowing the development of therapeutic strategies.

\section{From the editors' desks}

In 2017, when Apoptosis turned adult, we took over as editors of Apoptosis and Springer-Nature generously agreed to select new cover art for every volume [1,2]. Authors are welcomed to suggest or submit images of their own work. For previous volumes we selected impressive images provided by Drs. Malorni and Tinari [3] and Dr. Poon [4]. To adorn this volume we selected an image from a paper published in this issue of Apoptosis by Drs. Nowak-Sliwinska

Arjan W. Griffioen

a.griffioen@amsterdamumc.nl

$\triangle$ Patrycja Nowak-Sliwinska

Patrycja.Nowak-Sliwinska@unige.ch

1 Angiogenesis Laboratory, Department of Medical Oncology, Amsterdam UMC, Cancer Center Amsterdam, 1081 HV Amsterdam, The Netherlands

2 Faculty of Science, School of Pharmaceutical Sciences, University of Geneva, 1211 Geneva, Switzerland

3 Institute of Pharmaceutical Sciences of Western Switzerland, University of Geneva, 1211 Geneva, Switzerland

4 Translational Research Center in Oncohaematology, 1211 Geneva, Switzerland and Meraldi, showing multipolar cancer cell in late anaphase (Ducrey et al., current issue, https://doi.org/10.1007/s10495021-01671-3). Dividing cancer cell are characterized with an ability to "cluster" the poles of the mitotic spindle into a bipolar configuration. Those cells are prone to form multipolar spindles and to cluster their extra spindle poles to achieve pseudo-bipolar cell division.

The authors have previously shown that their optimized drug combination (ODC), containing four drugs, can target two distinct axes of cancer cell mitosis, while reducing the risk of acquired resistance and side effects. ODC acts on cancer cells, both with and without centrosome abnormalities, by inducing the formation of so called multipolar spindles. Furthermore, ODC inhibits spindle pole clustering of cancer cells. This, in turn, induces cell death in mitosis or in the following interphase, which is linked with severe chromosome mis-segregations [5].

\section{Discovery AhR ligand-new class of anti-cancer compounds}

The editors of Apoptosis are proud to announce the publication in this issue of Dr. Edmond Francis O'Donnel III from Dr. Siva Kolluri's group, on the discovery of an AhR ligand that induces apoptosis in cancer cells (current issue, https:// doi.org/10.1007/s10495-021-01666-0). The aryl hydrocarbon receptor $(\mathrm{AhR})$ is a ligand-activated transcription factor and a member of the bHLH/PAS (basic Helix-Loop-Helix/ Per-ARNT-SiM) family of proteins. Hypoxia inducible factor 1 (Hif-1) alpha and beta (also known as AhR Nuclear Translocator-Arnt) belong to the same family of proteins. Unlike Hif-1 alpha, which is regulated at the level of protein stability, AhR is a cytosolic ligand-activated transcription factor. Arnt serves as the common obligatory dimerization partner protein both for Hif- 1 alpha the AhR. The AhR was originally cloned as a receptor of dioxins, and has an evolutionary role in monitoring cellular stress and environmental insults. Recent data from Dr. Kolluri's laboratory and others indicate that the AhR has a tumor suppressive function 
in multiple cancers. The Kolluri laboratory hypothesized that Selective Modulators of AhR-regulated Transcription (SMAhRTs) can activate the tumor suppressive functions of the AhR. They embarked on a small molecule screen to identify SMAhRTs and systematically characterized their activity. In this issue of Apoptosis, O'Donnell et al. report their identification of CGS-15943 as a SMAhRT with potent anti-cancer activity in multiple cancer cell models. In addition to extensive characterization of the anti-cancer effects of this compound through the AhR signaling, O'Donnell et al. identified the downstream pathways that significantly contribute to the anti-cancer actions of CGS-15943. Thus, the study by O'Donnell et al. lays a solid foundation for developing SMAhRTs as a new class of anti-cancer compounds. Identification of cancers that responsive to AhR targeting and further elucidation of AhR-regulated tumor suppressive pathways in distinct cancers are exciting areas of research for the immediate future.

\section{The editors' choice}

The above mentioned growing interest in cell death mechanisms resulted in a number of high-profile papers published in Apoptosis. Cancer is a major field of research in cell death and different mechanisms of programmed cell death reported. A paper by Zhang et al. described pyroptosis as the chemotherapeutic effect of paclitaxel an d cisplatin in lung cancer cells (https://doi.org/10.1007/s10495-020-01648-8), while Shriwas et al. demonstrated that RNA helicase DDX3 plays an important role in resistance to cisplatin (https:// doi.org/10.1007/s10495-020-01591-8). Also in lung cancer, autophagy inhibition was demonstrated to revert chemotherapy resistance and to attenuate metastatic potential (https:// doi.org/10.1007/s10495-019-01526-y). In triple negative breast cancer it was shown that lactoferrin selectively induces apoptosis and cell cycle arrest (https://doi.org/10. 1007/s10495-019-01539-7). Oridonin and cetuxima b were reported to induce apoptosis and autophagy in laryngeal squamous cell carcinoma (https://doi.org/10.1007/s10495018-1497-0). A very interesting study showed that Klotho, a $\beta$-glucuronidase protein capable of hydrolyzing steroid $\beta$-glucuronides, can modulate ER crosstalk in non-cancerous cells between prosurvival authophagy and apoptosis (https://doi.org/10.1007/s10495-018-1496-1). Several studies focused on ischemic and cardiovascular diseases. Minocycline was found to promote cardiomyocyte mitochondrial autophagy to prevent cardiac dysfunction during sepsis (https://doi.org/10.1007/s10495-019-01521-3) and cardiac myocytes were found to undergo ER stress-dependent apoptosis by hyperosmotic conditions (https://doi.org/10.1007/ s10495-019-01558-4). Also excellent reviews were published on the cancer subjects of calreticulin in phagocytosis and cancer (https://doi.org/10.1007/s10495-019-01532-0) and heatshock proteins in melanoma (https://doi.org/10. 1007/s10495-019-01577-1), as well as in ischemic stroke (https://doi.org/10.1007/s10495-019-01556-6) and more mechanistical issues on the role of TFG-activated kinase-1 in cellular signals for death and survival (https://doi.org/10. 1007/s10495-018-1490-7).

It is for the team of editors and publishers of Apoptosis impossible to stress enough that a successful journal is fully dependent on the contributions of reviewers. We sincerely express gratitude to our editorial board and outside experts, whose work is an absolute requirement for the quality of a scientific journal. We also thank the authors who decided to have their papers published by Apoptosis. We, as the editors-in-chief of Apoptosis, will continue to improve the quality of the journal and serve the scientific community where possible.

\section{References}

1. Griffioen AW, Nowak-Sliwinska P (2017) Apoptosis turns 21. Apoptosis 22:1485-1486

2. Nowak-Sliwinska P, Griffioen AW (2018) Apoptosis on the move. Apoptosis 23:251-254

3. Griffioen AW, Nowak-Sliwinska P (2019) Cell death rocks. Apoptosis 24:205-207

4. Nowak-Sliwinska P, Griffioen AW (2020) Programmed death, cells on the last train to glory. Apoptosis 25:151-153

5. Weiss A et al (2019) Identification of a synergistic multi-drug combination active in cancer cells via the prevention of spindle pole clustering. Cancers 11(10):1612. https://doi.org/10.3390/ cancers 11101612

Publisher's Note Springer Nature remains neutral with regard to jurisdictional claims in published maps and institutional affiliations. 